\title{
Ressignificação da estética na contemporaneidade: Uma análise do perfil dos
}

\section{profissionais da área}

\author{
Resignification of contemporary aesthetics: An analysis of the profile of professionals in the field \\ Resignificación de la estética contemporánea: Un análisis del perfil de los profesionales en el campo
}

Recebido: 10/06/2021 | Revisado: 18/06/2021 | Aceito: 23/06/2021 | Publicado: 10/07/2021

\author{
Danielle Brangioni Pereira \\ ORCID: https://orcid.org/0000-0002-4747-1366 \\ Faculdade Dinâmica do Vale do Piranga, Brasil \\ E-mail: fisioderma@yahoo.com \\ Lúcia Meirelles Lobão Protti \\ ORCID: https://orcid.org/0000-0002-6775-9148 \\ Faculdade Dinâmica do Vale do Piranga, Brasil \\ E-mail: lucia.fadip@gmail.com \\ Emília Pio da Silva \\ ORCID: https://orcid.org/0000-0001-8130-5196 \\ Faculdade Dinâmica do Vale do Piranga, Brasil \\ E-mail: emiliapiosilva@yahoo.com.br \\ Marli do Carmo Cupertino \\ ORCID: https://orcid.org/0000-0001-5790-0622 \\ Faculdade Dinâmica do Vale do Piranga, Brasil \\ Universidade Federal de Viçosa, Brasil \\ E-mail: marli.cupertino@ufv.br
}

\begin{abstract}
Resumo
As revoluções nas áreas da tecnologia da informação e biotecnologia possibilitaram diferentes formas de interação, expressão e criação de subjetividade. Tanto mulheres, quanto homens, passaram a viver com grandes preocupações referentes aos cuidados pessoais, beleza e estética, buscando o corpo e o rosto perfeitos. Com isso, a área da estética tem sido cada vez mais reconhecida no âmbito profissional, atendendo aos anseios da maior parte da população, em relação à beleza e ao bem-estar. Assim, objetivou-se analisar, a partir da legislação vigente, o papel e as competências dos profissionais que podem atuar na área estética (fisioterapeuta, médico dermatologista e esteticista), na contemporaneidade. Para alcançar o objetivo proposto, realizou-se uma pesquisa documental das diretrizes curriculares do curso de fisioterapia e medicina, com especialização em dermatologia e estética. Houve um grande crescimento na área da estética nos últimos anos. Homens e mulheres passaram a se interessar pelo corpo perfeito e saudável, com aumento na demanda por profissionais capacitados na área. $O$ que difere a fisioterapia dermatofuncional, o médico dermatologista e o esteticista é o tempo de estudo e qualificações durante a graduação e pós-graduação. O fisioterapeuta atua de forma integrada com outros profissionais, auxiliando no tratamento de determinadas patologias do sistema tegumentar, enquanto o esteticista oferece aos clientes alternativas para melhorar sua aparência física, desde a limpeza da pele até cuidados pós-cirúrgicos. O médico dermatologista é a especialidade tanto clínica, quanto cirúrgica. Conclui-se a fisioterapia dermatofuncional, o médico dermatologista e a estética, são profissões com responsabilidades e competências diferenciadas, ambas em franca expansão de demanda.
\end{abstract}

Palavras-chave: Fisioterapia; Dermatologia; Estética; Saúde pública.

\begin{abstract}
The revolutions in the area of information technology and biotechnology made possible different forms of interaction, expression and creation of subjectivity. Both women and men start to live with great concern regarding personal care, beauty and aesthetics, seeking the perfect body and face. With this, the area of aesthetics has been increasingly recognized in the professional sphere, meeting the desires of most of the population in relation to beauty and wellbeing. Thus, the objective was to analyze, based on current legislation, the role and skills of professionals who can work in the esthetic area (physiotherapist, dermatologist and beautician) in contemporary times. To achieve the proposed objective, a documental research of the Curriculum Guidelines of the Physiotherapy and Medicine course with specialization in dermatology and aesthetics was carried out. There has been a huge growth in the area of aesthetics in recent years. Men and women became interested in the perfect and healthy body, with an increase in the demand for trained professionals in the area. What differentiates dermatofunctional physiotherapy, the dermatologist and the beautician is the study time and qualifications during undergraduate and graduate studies. The physiotherapist works in an integrated way with other professionals, helping in the treatment of certain pathologies of the integumentary system, while the beautician offers clients alternatives to improve their physical appearance, from skin
\end{abstract}


cleaning to post-surgical care. The dermatologist is both a clinical and surgical specialty. It is concluded that dermatofunctional physiotherapy, the dermatologist and esthetics are professions with different responsibilities and competences, both in clear expansion of demand.

Keywords: Physiotherapy; Dermatology; Aesthetics; Public health.

\begin{abstract}
Resumen
Las revoluciones en el área de las tecnologías de la información y la biotecnología posibilitaron diferentes formas de interacción, expresión y creación de subjetividad. Tanto mujeres como hombres viven con gran preocupación por el cuidado personal, la belleza y la estética, buscando el cuerpo y el rostro perfectos. Con ello, el área de la estética ha sido cada vez más reconocida en el ámbito profesional, satisfaciendo los deseos de la mayoría de la población en relación a la belleza y el bienestar. Así, el objetivo fue analizar, a partir de la legislación vigente, el papel y las competencias de los profesionales que pueden trabajar en el área de la estética (fisioterapeuta, dermatólogo y esteticista) en la época contemporánea. Para lograr el objetivo propuesto, se llevó a cabo una investigación documental de las Directrices Curriculares del curso de Fisioterapia y Medicina con especialización en Dermatología y estética. Ha habido un gran crecimiento en el área de la estética en los últimos años. Hombres y mujeres se interesaron por el cuerpo perfecto y saludable, con un aumento en la demanda de profesionales capacitados en el área. Lo que diferencia a la fisioterapia dermatofuncional, la dermatóloga y la esteticista, es el tiempo de estudio y la titulación durante los estudios de pregrado y posgrado. El fisioterapeuta trabaja de forma integrada con otros profesionales, ayudando en el tratamiento de determinadas patologías del sistema tegumentario, mientras que la esteticista ofrece a sus clientes alternativas para mejorar su apariencia física, desde la limpieza de la piel hasta el cuidado posquirúrgico. El dermatólogo es una especialidad tanto clínica como quirúrgica. Se concluye que la fisioterapia dermatofuncional, el dermatólogo y la estética son profesiones con responsabilidades y competencias distintas, ambas en clara expansión de demanda.
\end{abstract}

Palabras clave: Fisioterapia; Dermatología; Estética; Salud pública.

\title{
1. Introdução
}

Na contemporaneidade, a concepção corporal feminina tem sido vinculada a normas, que variam de acordo a padrões estéticos estipulados pelas mídias digitais. Com o advento das redes sociais, as mulheres têm aderido a padrões estéticos flexibilizados, capazes de serem transformar de acordo com as exigências vigentes. Padrões corporais específicos têm adquirido significado de aceitação ou rejeição social, associando o corpo perfeito ao sucesso (Lira, Ganen, Lodi \& Alvarenga, 2017; Campos, Faria \& Sartori, 2020).

O Brasil é o terceiro país no ranking mundial no mercado de beleza, ficando atrás apenas da China e dos Estados Unidos. Apesar da queda de 6,7\% nas vendas do setor entre 2014 e 2015, o brasileiro ainda destina 2\% do orçamento aos cuidados com a estética e higiene pessoal, segundo a Associação Brasileira da Indústria de Higiene Pessoal, Perfumaria e Cosméticos (ABIHPEC, 2017). De acordo com a Sociedade Brasileira de Cirurgia Plástica (SBCP), em 2018 foram registradas mais de 1 milhão 498 mil cirurgias plásticas estéticas no Brasil. Esses números fizeram com que o país permanecesse em primeiro lugar no ranking mundial de países que mais realizam cirurgias plásticas, sendo a lipoaspiração, rinoplastia e próteses mamárias as intervenções estéticas mais comuns (SBCP, 2020).

Desde a antiguidade, a beleza do indivíduo tem sido associada a características de saúde e felicidade. Porém, ser belo não deve significar assemelhar-se a alguém ou estar dentro de um padrão estabelecido socialmente, mas sim, sentir-se satisfeito com a sua própria aparência (Freitas, 2010). Atualmente, a preocupação com a imagem corporal tem aumento, devido ao fato de a beleza ter adquirido um status de aceitação e/ou rejeição social. A preocupação com padrões estéticos tem atingido níveis excessivos, principalmente, após o advento das mídias sociais e a inúmeras propagandas que associam o corpo perfeito ao sucesso. As mídias têm imposto um padrão de beleza cada vez mais difícil de ser atingido, impulsionando os indivíduos a viverem uma procura incessante pela perfeição corporal, por meio de dietas, exercícios físicos, fármacos, cirurgias plásticas e outros procedimentos estéticos. O corpo feminino tem grande valor e influência, tornando-o alvo de cobrança para o posicionamento da mulher no padrão social. O autocontrole, gera um olhar social crítico, como a fiscalização da autoimagem e o empenho para adequar o corpo à norma (Russo, 2005; Campos, Faria \& Sartori, 2020). 
Desde a última década do século passado, tem-se observado um aumento do interesse dos profissionais da área de fisioterapia pelo tratamento de distúrbios metabólicos, além de alterações físicas, estéticas e funcionais. Tal interesse, fez com que surgisse uma especialidade primeiramente denominada de fisioterapia estética, a qual tinha por objetivos melhorar ou restaurar a aparência corporal e facial, mas, que em virtude das diversas áreas e campos de atuação do fisioterapeuta e dos recursos que esses profissionais têm a sua disposição, passou a ser denominada de fisioterapia dermatofuncional (Milani, João $\&$ Farah, 2006).

Apesar de haver semelhanças em procedimentos nas profissões de fisioterapia dermatofuncional, estética e medicina com especialização em dermatologia, são profissões com abordagens diferenciadas. Compreendendo a importância de cada uma delas, cada qual na sua esfera de ação, buscou-se responder ao seguinte questionamento: quais as competências específicas da fisioterapia dermatofuncional, da dermatologia como especialidade médica, e da estética?

A fisioterapia dermatofuncional, estética e medicina dermatológica são profissões com responsabilidades e competências diferenciadas, porém com os mesmos propósitos. O que difere essas profissões são as diretrizes para o curso de graduação, o tempo de estudo e qualificações. Nesse ponto, é essencial destacar que a presente pesquisa surgiu de questionamentos, muitas vezes levantados por acadêmicos destas áreas, e pode contribuir para o reconhecimento do verdadeiro papel de cada profissional envolvido. Assim, objetivou-se analisar, a partir da legislação vigente, o papel e as competências dos profissionais que podem atuar na área estética (fisioterapeuta, médico dermatologista e esteticista).

\section{Metodologia}

A pesquisa realizada caracterizou-se como uma pesquisa documental, qualitativa (Estrela, 2018), feita a partir da análise das Diretrizes Curriculares Nacionais $(\mathrm{DCN})$ dos cursos de graduação de profissionais que trabalham na área estética. Na medicina trata-se da DCN com o Parecer CNE/C n. 116/2014; para Estética a Resolução CNE/CES n. 5, de 16 de novembro de 2016, regulamentada no dia 03 de abril de 2018 pelo MEC; e da Fisioterapia, a resolução CNE/CSE n. 4, de 19 de fevereiro de 2002.

Adicionalmente foi feita uma revisão de literatura realizada nas bases eletrônicas de dados LILACS, SCIELO e PUBMED/Medline com busca por artigos publicados no período de 2010 a 2020. Foi feita a busca por artigos originais com os descritores de "Fisioterapia" AND "Dermatologia", "Fisioterapia" AND "Estética", publicados na língua portuguesa e inglesa, com abordagem na: I) a estética na contemporaneidade e/ou; II) o perfil dos profissionais da área estética.

\section{Resultados e Discussão}

No Brasil, a busca por procedimentos com fins estéticos contabilizou um aumento significativo na última década (Lira et al., 2017; Campos et al., 2019), ampliando a demanda por profissionais cada vez mais qualificados para atuarem na área estética. Além do conhecimento técnico, tem aumentado a demanda por profissionais que possuem uma formação holística, com conhecimento, além das condições físicas do paciente e técnicas necessárias ao procedimento, entender também as demandas psicossociais e socioafetivos. Tal fato é devido à mudança do padrão dos indivíduos, que buscam por procedimentos estéticos. Alguns deles possuem outros distúrbios, que podem estar associados a uma ausência de identificação da própria da beleza (Campos et al., 2019).

$\mathrm{Na}$ contemporaneidade, tem surgido patologias como o transtorno dismórfico corporal, que é definido como uma preocupação exagerada com um ou mais defeitos inexistentes, ou sutis, da aparência. Tal transtorno pode resultar em procedimentos desnecessários ocasionando efeitos colaterais catastróficos. Porém, se não forem realizados, resultam em forte 
angústia ou prejudicam a capacidade funcional do indivíduo, necessitando de profissionais capacitados para atender e diagnosticar tais transtornos. (Gracindo, 2015).

As revoluções nssa área da tecnologia da informação e biotecnologia possibilitaram diferentes formas de interação, expressão e criação de subjetividade. O instagram, por exemplo, é uma rede que mais cresce atualmente, possibilitando cerca de 1 bilhão de usuários ativos, sendo $75 \%$ mulheres onde os padrões de beleza prevalecem aos cuidados com o corpo perfeito (Cruz, Ueno \& Manzano, 2015).

No panorama nacional, pesquisas revelam que o público feminino não é satisfeito esteticamente em cerca de 18 a 83 $\%$, e o público masculino cerca de 2 a 73\%. Tal insatisfação apresenta crescimento devido a influencia dos meios de comunicação, que pressionam de maneira subliminar ao oferecerem e colocarem em evidência procedimentos estéticos bem sucedidos (Pinheiro, 2020).

Com o desenvolvimento da tecnologia, tanto mulheres quanto homens, vivem com grande preocupação referente aos cuidados pessoais, beleza e estética, buscando o corpo e o rosto perfeitos. Com isso, a área da estética tem sido cada vez mais reconhecida no âmbito profissional, atendendo aos anseios da maior parte da população em relação à beleza e ao bem-estar (Cruz, et al., 2015).

Historicamente, a estética surgiu ligada a cosmetologia, conectando a cosmética a práticas religiosas, prevalecendo ao longo da história antiga. Os primeiros profissionais ligados a estética e a beleza do corpo, surgiram na Grécia, sendo assim chamados de Kosmetes. Nesta época eram os escravos responsáveis por todo o cuidado estético, sendo assim chamados hoje de esteticistas. Foi em Roma que surgiu o primeiro colégio profissional, denominado, Collegium Aromatorium, mas com a queda do Império Romano a cosmética teve uma queda, ressurgindo apenas em 1880, passando a contar com uma grande quantidade de técnicas e tratamentos. Ao longo da história veio a necessidade de profissionalizar o esteticista, o qual era responsável por cuidar da beleza do ser humano de todas as classes sociais (Gonçalves, 2006).No Brasil, conforme explicam Schmitz, Laurentino e Machado (2012), a história dos profissionais da estética tem seu início na década de 60, por meio de Anne Marie Klotz, filha de um diplomata francês, que, tendo aprendido o ofício na França, criou a primeira escola de Estética no Rio de Janeiro, a escola France Bel e Federação Brasileira de Estética e Cosmetologia (FEBECO).

Hoje, no campo da saúde, a construção dos sentidos e valores sobre a estética estão cada vez mais presentes influenciando na construção da identidade do indivíduo e na percepção que esse tem de si mesmo. Desta forma, enfatiza-se que é necessário um profissional que seja qualificado, atualizado e preparado para transmitir segurança e garantir a eficácia do serviço oferecido (Normando, 2012). Apesar de o esteticista atender e cuidar de seus pacientes, ainda que esta categoria não apresente formação médica, fica claro a necessidade de certificação para o desenvolvimento dos procedimentos.

Para atuar em um mercado de trabalho em expansão, como a área estética, é necessário que o profissional em cosmetologia e estética estejam capacitados. O profissional de estética atua nas seguintes áreas: estética facial, estética corporal e estética capilar. Sendo que os tratamentos faciais são realizados para melhorar, principalmente, de atributos relacionados a hidratação e pigmentação da pele, causada por fatores bióticos e abióticos. E no quesito corporal, destacam-se tratamentos visando atenuar a perda do colágeno e elasticidade da pele, bem como melhora no aspecto capilar (Schmitz, et al. 2012).

\subsection{Avaliação das competências profissionais}

$\mathrm{Na}$ área estética, destacam-se três profissionais de atuação relevante. Trata-se do fisioterapeuta com especialidade dermatofuncional, o médico especialista em dermatologia e o esteticista. A análise das habilidades e competências de cada profissão podem ser feitas através das DCNs de cada curso. Conforme é discutido a seguir. 
O Fisioterapeuta e o Esteticista, apesar de trabalharem com os assuntos e alguns procedimentos parecidos, estes profissionais possuem abordagem diferentes. Um esteticista é habilitado para realizar tratamentos necessários para resolver a maioria dos problemas estéticos. Já o fisioterapeuta dermatofuncional oferece recursos que consistem nos tratamentos estéticos, pela manutenção da integridade do sistema tegumentar como um todo, incluindo as suas estruturas relacionadas. Desta forma a sua atuação é ampla e diversa atingindo várias áreas.

O profissional fisioterapeuta dermatofuncional além dos seus conhecimentos de anatomia, fisiologia, patologia e cosmetologia, dispõe de meios físicos e naturais, modelos, métodos e técnicas terapêuticas, que permite reabilitar eficientemente diversas patologias clínica do foro estético, proporcionando uma abordagem terapêutica de forma completa, potenciando e assegurando resultados efetivos. Então podemos dizer que a esteticista cuida do embelezamento da pele, e isso requer pele e seus anexos, ou seja, capilar, facial e corporal. A dermatofuncional além de trabalhar na área da estética, ela cuida da reabilitação, e recuperação do sistema tegumentar uma abordagem funcional.

A respeito da dermatologia, como especialidade médica, ela atua em todos os processos fisiopatológicos que envolvem a pele: desde simples infecções, reações autoimunes e inflamatórias além de tumores, dermocosmiatria e procedimentos cirúrgicos de maior complexidade da pele e seus anexos. Essa especialidade é certificada pelo médico sendo registrado junto ao Conselho Federal de Medicina (CFM) e registrada pela Sociedade Brasileira de Dermatologia.

\subsubsection{Fisioterapia}

As Diretrizes Curriculares Nacionais do Curso de Graduação em Fisioterapia foram criadas em 19 de fevereiro de 2002, com o objetivo de romper com o foco na doença e avançar para a concepção ampliada de saúde, definidas a partir das competências, habilidades gerais e específicas para o perfil profissional, generalista, humanista, crítico e reflexivo aptos a desenvolver ações em todos os níveis de atenção à saúde. A partir dessa concepção os profissisonais envolvidos, foram estruturados em seis categorias: atenção à saúde, tomada de decisões, comunicação, liderança, administração e gerenciamento e educação permanente. (Bertoncello, Pivetta, 2015). Também é importante destacar que estão descritas na Res. CNE/CES n. 4/2002 as competências e habilidades específicas do núcleo da Fisioterapia, seguidas dos conteúdos curriculares e dos demais artigos que abordam questões referentes aos estágios, trabalho de conclusão de curso, projeto pedagógico de modo geral, entre outros.

De acordo com Bertoncello e Pivetta (2015), o profissional de Fisioterapia está capacitado para atuar em nas distintas subáreas do conhecimento, sendo elas, hospitais, clínicas, ambulatórios, consultórios, centros de reabilitação, programas instituicionais, ações básicas de saúde, fisioterapia do trabalho, indústria de equipamento de uso fisioterapêutico, pesquisa, supervisão e docência.

As DCNs para o curso de graduação em Fisioterapia trazem orientações para reafirmar avanços no âmbito técnico, científico, tecnológico, pedagógico, conceituais, epidemiológico, sócio-demográfico e legais na profissão. Complementando, Bertoncello e Pivetta (2015, p. 78) afirmam que existem questões relevantes que não foram internalizadas em relação à DCNs, como "a dicotomia teoria prática que ainda prevalece em alguns projetos de curso, a formação distante da realidade de saúde da população e o não atendimento às demandas de saúde do país". Atualmente as DCNs que regem o curso de fisioterapia são do ano de 2002 ainda a se parear as lacunas identificadas nas DCN atuais, colocando-se em questão o debate sobre os possíveis alinhamentos que se faz necessário. (Bertoncelo \& Pivetta, 2015).

A fisioterapia dermatofuncional é uma área da fisioterapia que atua, principalmente, em patologias relacionadas ao tegumento. Busca comprovação científica dos métodos e técnicas utilizados para o tratamento de diversas patologias (Guirro \& Guirro, 2003). Para preparar o protocolo para um tratamento, o fisioterapeuta dermatofuncional analisa peso, medidas e hábitos de vida e alimentares dos pacientes. Essa avaliação determina os recursos terapêuticos, a duração e a frequência do tratamento. 
Dessa forma, cada paciente é estudado e o método a ser tratado é individualizado (Oliveira, 2019). As competências do profissional dermatofuncional consulta, solicita e, avalia , determina o diagnóstico e prognóstico fisioterapêutico, executa recursos terapêutico manuais, utilizando recursos de ação isolada como termoterapêutico, crioterapêutico, eletroterapêutico, promovendo a recuperação tegumentar, no que se refere aos distúrbios endócrino-metabólico, dermatológico, linfático, circulatório, osteomioarticular, como, as disfunções de queimaduras, hanseníase, dermatose, psoríase, vitiligo, cicatrizes aderentes, hipertróficas, queloídeanas, obesidade, gordura localizada, linfedema, fleboedemas, estrias atróficas entre outras. Ainda é de sua competência promover e recuperar o pré e pós-operatório de cirurgia plástica reparadora e bariátricas (Brasil, 2014).

O profissional fisioterapeuta vem se destacando nos últimos anos na área estética, principalmente pela formação holística que possui, devido a especialização e aperfeiçoamento profissional. Entretanto, estudos que investiguem o perfil do profissional que atua na área estética, incluindo médicos dermatologistas e esteticistas, além da capacitação do profissional fisioterapeuta para atuar na área estética são escassos. Os profissionais fisioterapeutas precisam conhecer mais profundamente seu campo de atuação tendo como base a legislação vigente a fim de reivindicar competências privadas à área. Além disso, deve fazer-se conhecido as DCNs para um melhor embasamento de nossos recursos. Outro fato que nos chama atenção é que na prática clínica muitos e até mesmo os acadêmicos desconhecem a diferenciação dos profissionais Esteticistas, Dermatologista e Fisioterapeutas, dificultando assim funções que é exclusivamente do Fisioterapeuta (Borges, 2016).

A contemporaneidade demanda uma educação que estimule os estudantes a pensar com autonomia e reflexão crítica. Ao mesmo tempo, mudanças na formação profissional em fisioterapia foram necessárias para que os currículos atendessem às necessidades específicas das regiões, às expectativas dos docentes e discentes e incluíssem a tríade ensino, pesquisa e extensão. Assim, foram elaboradas as DCNs. Outrossim, as IES precisam avaliar o clima organizacional que permite conhecer o ambiente de trabalho e o nível de qualidade praticado nessas instituições.

O COFFITO, por meio da portaria n. 45, de 11 de fevereiro de 2016, instaurou a comissão de análise e proposta de reformulação das DCNs da fisioterapia para que possa desencadear o processo de estudo e revisão das DCNs vigentes no Brasil. O grupo conta com representantes da Associação Brasileira de Ensino em Fisioterapia (ABENFISIO), elaborando uma nova proposta que complete as necessidades da profissão e da sociedade.

\subsubsection{Esteticista}

O primeiro curso de estética foi fundado no Rio de Janeiro (RJ), por Anne Marie Koltz na década de 50, surgindo em 2003 o projeto da Lei $\mathrm{n}^{\mathrm{a}} 959$ no qual o profissional esteticista poderia ter uma formação a nível superior. A regulamentação aprova a Lei 13.643 formatizando o profissional como Esteticista e Cosmetológo. Essa profissão inclui várias habilitações, com carga horária mínima de 180 horas, pós-graduação interdisciplinar dentro da área da sáude, entre outras. Porém, observase que ainda existe falta de conhecimento sobre a evolução das atividades realizadas atualmente em relação à função desse profissional, tratando-se de um instrumento fundamental para o conhecimento do perfil dos graduandos (Fogliatto, Mioto \& Gonçalves 2018).

Para se profissionalizar como esteticista, exigem-se curso técnico com concentração em Estética ou curso de nível superior com concentração em Estética e Cosmética, ou equivalente. O profissional da estética ele tem uma formação de tecnólogo, reconhecido pelo MEC, tendo uma duração de três a quatro anos completamente voltados para a estética. O foco na área da estética é capilar, facial e corporal, ou seja, é baseado apenas para fins estéticos (Brasil, 2018).

O esteticista é o profissional que atua na melhora fisiológica da pele, por meio de tratamentos e cosméticos específicos, atuando de forma relevante na área da saúde preventiva. No Brasil, por meio da Lei n. 13.643/18, ficou 
regulamentada a profissão de Esteticista, compreendendo o Esteticista e Cosmetólogo, e de Técnico em Estética (Brasil, 2018). Como funções do esteticista, a supracitada Lei aponta:

Executar procedimentos estéticos faciais, corporais e capilares, utilizando como recursos de trabalho produtos cosméticos, técnicas e equipamentos com registro na Agência Nacional de Vigilância Sanitária (ANVISA); solicitar, quando julgar necessário, parecer de outro profissional que complemente a avaliação estética e observar a prescrição médica ou fisioterápica apresentada pelo cliente, ou solicitar, após exame da situação, avaliação médica ou fisioterápica. (Brasil, 2018, art. 5).

\subsubsection{Médico Dermatologista}

O médico dermatologista é responsável pelo diagnóstico, prevenção ou tratamento das doenças e problemas que atingem a pele e seus anexos. O dermatologista não somente cresceu, mas, transformou-se. De especialidade puramente clínica, tornou-se especialidade médico-cirúrgica. Além disso, foi crescendo em uma nova área de atuação, a dermatologia cosmética, que desperta enorme interesse da coletivamente e da mídia (Fogliatto, et al., 2018)

As DCNs dos cursos de graduação médica, nas últimas décadas, buscam garantir uma formação ampliada e em consonância com as demandas atuais no sistema de saúde brasileiro. O ensino de certos componentes do conhecimento clínico ainda é desafiante, principalmente na especialidade da Dermatologia. As DCNs do curso de graduação em Medicina, emitidas pelo Conselho Nacional de Educação (CNE) buscaram mudar a prevalência dessas características visando à capacitação do médico dermatologista nos diferentes níveis de atenção, especialmente primário e secundário, fomentando a aptidão desses profissionais para promover, recuperar e reabilitar a saúde, a fim de prevenir agravos (Brasil, 2014).

O médico dermatologista deverá aprender e praticar nos três anos para a sua formação na especialidade. Os programas eram simples e compostos apenas pela realização do prazo e setores de rodízio de cada um deles. Um dos principais benefícios da reformulação da matriz em 2006, foi deixar claro que o médico com residência em dermatologia está capacitado para atuar nas áreas clínica, cirúrgica, e sanitária, assim como nos segmentos de cosmiatria e estética. No decorrer dos três anos de especialização as suas competências se tornam como propósito consolidar mais conhecimentos, habilidades e atitudes fazendo com que o especialista na área médica em dermatologia, acrescente nas suas competências casos clínico-cirúrgicos dermatologicamente mais avançados (SBD, 2019).

As mais recentes DCNs do curso de graduação em Medicina foram homologadas pelo Conselho Nacional de Educação por meio da Resolução n. 3, de 20 de junho de 2014. As diretrizes específicas da área de dermatologia, têm como objetivo alinhar metas com ênfase na atualização científica para a melhoria das condições de assistência à população, tendo atuação no atendimento das doenças de pele e a promoção de uma dermatologia abrangente e acessível além dos consensos de hidradenite supurativas, urticária, dermatite atópica, e doenças bolhosas, além das diretrizes de psoríase (SBD, 2019).

O Quadro 1 traz a síntese das competências dos três profissionais: fisioterapeuta Dermatofuncional, Esteticista e Dermatologista. 


\begin{tabular}{|c|c|c|c|}
\hline \multicolumn{4}{|c|}{ Quadro 1 - Síntese das competências de cada profissão. } \\
\hline & FISIOTERAPIA & MEDICINA & ESTÉTICA \\
\hline REGULAMENTAÇÃO & $\begin{array}{l}\text { A Resolução CNE/CSE n. 4, } 19 \text { de fevereiro de } \\
2002 .\end{array}$ & Resolução CNE/C n. 116,13 de abril de 2014. & A Resolução CNE/CES n. 5, 3 de abril de 2018. \\
\hline DURAÇÃO & $\begin{array}{l}\text { Carga horária mínima de } 4.000 \text { horas com duração } \\
\text { do curso em } 5 \text { anos. }\end{array}$ & $\begin{array}{l}\text { Carga horária mínima de } 7.200 \text { (sete mil e } \\
\text { duzentas) horas e prazo mínimo de } 6 \text { (seis) anos } \\
\text { para sua integralização. }\end{array}$ & $\begin{array}{l}\text { Duração de } 3 \text { anos para o tecnólogo e o } \\
\text { Bacharelado em Estética e Cosmetologia } \\
\text { consiste no tempo de } 4 \text { anos. }\end{array}$ \\
\hline Perfil do Egresso & $\begin{array}{l}\text { Generalista, humanista, crítica e reflexiva, } \\
\text { capacitado a atuar em todos os níveis de atenção à } \\
\text { saúde, com base no rigor científico e intelectual. } \\
\text { Detém visão ampla e global, respeitando os } \\
\text { princípios éticos/bioéticos, e culturais do indivíduo } \\
\text { e da coletividade. Capaz de ter como objeto de } \\
\text { estudo o movimento humano em todas as suas } \\
\text { formas de expressão e potencialidades, quer nas } \\
\text { alterações patológicas, cinético-funcionais, quer } \\
\text { nas suas repercussões psíquicas e orgânicas, }\end{array}$ & $\begin{array}{l}\text { Generalista, humanista, crítica, reflexiva e ética, } \\
\text { com capacidade para atuar nos diferentes níveis e } \\
\text { todas áreas de atenção à saúde. }\end{array}$ & $\begin{array}{l}\text { Capacitado a atuar nas áreas de prestação de } \\
\text { serviços estéticos da região fácil, corporal e } \\
\text { capilar, capacitado ao uso correto das técnicas e } \\
\text { dos equipamentos utilizados nos procedimentos } \\
\text { estéticos }\end{array}$ \\
\hline Competências gerais & $\begin{array}{l}\text { O curso objetiva formar um profissional } \\
\text { capacitado a preservar, desenvolver, restaurar a } \\
\text { integridade de órgãos, sistemas e funções, desde a } \\
\text { elaboração do diagnóstico físico e funcional, } \\
\text { eleição e execução dos procedimentos } \\
\text { fisioterapêuticos pertinentes a cada situação. }\end{array}$ & $\begin{array}{l}\text { O curso capacita o aluno a promoção, prevenção, } \\
\text { recuperação e reabilitação da saúde, nos âmbitos } \\
\text { individual e coletivo, com responsabilidade social } \\
\text { e compromisso com a defesa da cidadania, da } \\
\text { dignidade humana, da saúde integral do ser } \\
\text { humano e tendo como transversalidade em sua } \\
\text { prática, sempre, a determinação social do } \\
\text { processo de saúde e doença. }\end{array}$ & $\begin{array}{l}\text { O curso objetiva proporcionar ao aluno } \\
\text { conhecimentos teóricos científicos atualizados } \\
\text { que preparem o aluno para atuação nos diversos } \\
\text { da área. Atuação nos diversos segmentos de } \\
\text { prestação de serviço, tanto facial, quanto } \\
\text { corporal e capilar, desde o atendimento à } \\
\text { clientela até a área de cosmetologia e saúde, de } \\
\text { modo a permitir o uso correto das técnicas e dos } \\
\text { equipamentos utilizados nos procedimentos } \\
\text { estéticos, de forma preventiva }\end{array}$ \\
\hline $\begin{array}{l}\text { Competências específicas } \\
\text { da área dermatofuncional }\end{array}$ & $\begin{array}{l}\text { Realizar consulta fisioterapêutica, anamnese, } \\
\text { solicitar e realizar interconsulta e } \\
\text { encaminhamento, realizar avaliação } \\
\text { cinésiofuncional e física, determinar diagnóstico e } \\
\text { prognóstico, prescrever e executar recursos } \\
\text { terapêuticos, prevenir, promover e realizar a }\end{array}$ & $\begin{array}{l}\text { As Diretrizes específicas da área de dermatologia, } \\
\text { tem como objetivo alinhar metas com ênfase na } \\
\text { atualização científica para a melhoria das } \\
\text { condições de assistência à população tendo } \\
\text { atuação nos atendimentos das doenças de pele e a } \\
\text { promoção de uma dermatologia abrangente e }\end{array}$ & $\begin{array}{l}\text { O curso de Estética tem como objetivo } \\
\text { proporcionar ao aluno conhecimentos teóricos } \\
\text { científicos atualizados que preparem o aluno } \\
\text { para atuação nos diversos da área. Atuação nos } \\
\text { diversos segmentos de prestação de serviço, } \\
\text { tanto facial, quanto corporal e capilar, desde o }\end{array}$ \\
\hline
\end{tabular}


recuperação do sistema tegumentar no que se refere aos distúrbios endócrino, metabólico,

dermatológico, linfático, circulatório,

osteomiarticular e neurológico como as disfunções

de queimaduras, hanseníase, dermatoses, psoríase,

vitiligo, piodermites, acne, cicatrizes aderentes,

cicatrizes hipertróficas, queloídeanas, úlceras

cutâneas, obesidade, adiposidade localizada,

envelhecimento, estrias atróficas, hipertricose,

linfoedemas, entre outras funcionalidades ou

estética. Além de prevenir, promover e realizar a

atenção fisioterapêutica pré e pós-operatória de

cirurgias plásticas reparadoras e bariátricas. acessível além dos consensos de hidradenite supurativas, urticária, dermatite atópica, e doenças

bolhosas, além das diretrizes de psoríase. atendimento à clientela até a área de

cosmetologia e saúde, de modo a permitir o uso correto das técnicas e dos equipamentos

utilizados nos procedimentos estéticos, de forma preventiva.

Fonte: Autores. 


\section{Considerações Finais}

Houve um grande crescimento na área da estética nos últimos anos. Homens e mulheres passaram a se interessar pelo corpo perfeito e saudável, com aumento na demanda por profissionais capacitados na área.

A literatura aponta que a fisioterapia dermatofuncional, o médico dermatologista e a estética, são profissões com responsabilidades e competências diferenciadas. O que difere essas três profissões é o tempo de estudo e qualificações durante a graduação e pós-graduação. Por isso os profissionais da área estética possuem competências diferenciadas de acordo com a formação que cada um recebeu.

O fisioterapeuta atua de forma integrada com outros profissionais, auxiliando no tratamento de determinadas patologias do sistema tegumentar, enquanto o esteticista oferece aos clientes alternativas para melhorar sua aparência física, desde a limpeza da pele até cuidados pós-cirúrgicos. O médico dermatologista é a especialidade tanto clínica, quanto cirúrgica.

Devido as alterações nos cenários da tecnologia da informação e biotecnologia, houve ressignificação dos padrões estéticos, tornando necessários estudos futuros buscando por ferramentas e estratégias para ressignificar, também, os profissionais na área. Estudos visando a formação humanística, pautada na bioética adquiriram importância semelhante ao estudo de técnicas e procedimentos ligados a questões físicas. Tal fato se deve ao aparecimento de patologias ligadas a estética, como o transtorno dismórfico corporal, necessitando de pesquisas que visem o desenvolvimento de habilidades e competências para o tratamento de questões socioafetivas e psicossociais dos pacientes.

\section{Agradecimentos}

Os autores agradecem o apoio da Faculdade Dinâmica do Vale do Piranga (FADIP).

\section{Referências}

ABIHPEC. Associação Brasileira da Indústria de Higiene Pessoal, Perfumaria e Cosméticos. (2017). Mercado. https://abihpec.org.br/mercado/

Bertoncello, D. \& Pivetta, H. M. F. (2015). Diretrizes Curriculares Nacionais para a Graduação em Fisioterapia: Reflexões Necessárias. Cad Edu Saude e Fis. 2(4). http://revista.redeunida.org. br.

Borges, K. P. (2016). A Fomarção do Fisioterapeuta na perspectiva das diretrizes curriculares nacionais e das competências no âmbito da promoção da saúde. 179f. Tese (Doutorado em Psicologia Clínica e Cultura) - Universidade de Brasília, 2016. https://repositorio.unb.br.

Brasil. (2018). Lei $n^{\circ} 13.643$, de 3 de abril de 2018. Regulamenta as profissões de Esteticista, que compreende o Esteticista e Cosmetólogo, e de Técnico em Estética. https://legislação.presidencia.gov.br".

Brasil. Ministério da Educação. (2014). Diretrizes Curriculares Nacionais do Curso de Graduação em Medicina. RESOLUÇÃO CNE/C nº116,13 abril de 2014. http://www.abmes.org.br > public > legislação > parece

Brasil. Ministério da Educação. (2018). Matriz De Competências - Dermatologia. https://www.sbd.org.br >notícias >mec-aprova-matriz

Campos, R. G., Faria, H. M. C. \& Sartori, I. D. (2019) Cultura da estética: O impacto do instragram na subjetividade feminina. Caderno de psicologia, 1(2), 310-334, 2019. https://seer.cesjf.br/index.php/cadernospsicologia/article/view/2495.

CNE. Conselho Nacional de Educação. (2002). Resolução $n^{\circ}$ CNE/CES 4, de 19 de fevereiro de 2002. Institui Diretrizes Curriculares Nacionais do Curso de Graduação em Fisioterapia. http://www.crefito10.org.br/conteudo.jsp?ids=24.

COFFITO. Conselho Federal de Fisioterapia e Terapia Ocupacional. (2002). RESOLUÇÃO No CNE/CES 4, de 19 de fevereiro de 2002. Institui Diretrizes Curriculares Nacionais do Curso de Graduação em Fisioterapia. http://www.crefito10.org.br/conteudo.jsp?ids=24.

COFFITO. Conselho Federal de Fisioterapia e Terapia Ocupacional. (2021). Definição Fisioterapia. https://coffito.gov.br

Cruz, J. C. R., Ueno, N. F. \& Manzano, B. M. (2015). O Estudo Científico como Base na Área da Estética: uma Contrapartida ao Senso Comum. Revista Científica da FHO|UNIARARAS, 3(2), 2.

Estrela, C. (2018). Metodologia Científica: Ciência, Ensino, Pesquisa. Editora Artes Médicas. 738 p.

Fogliatto, P. E., Mioto, M. B. M. \& Gonçalves, V. P. (2018). Artigo Científico - Perfil Profissional dos Egressos do curso de Cosmetologia e Estética UNISUL/PB. p. 2. https://www.riuni.unisul.br

Freitas, C. M. S. M. de, Lima, R. B. T., Costa, A. S., \& Lucena Filho, A. (2010). O padrão de beleza corporal sobre o corpo feminino mediante o IMC. Revista Brasileira De Educação Física E Esporte, 24(3), 389-404. https://doi.org/10.1590/S1807-55092010000300010 
Research, Society and Development, v. 10, n. 8, e16510817045, 2021

(CC BY 4.0) | ISSN 2525-3409 | DOI: http://dx.doi.org/10.33448/rsd-v10i8.17045

Gonçalves, Á. (2006) Manual Técnico de Estética - Teoria e Prática para Estética, Cosmetologia e Massagem. Ondagrafe. 275 p.

Gracindo, L. C. (2015) A moralidade das intervenções cirúrgicas com fins estéticos de acordo com a bioética principialista. Rev. Bioét., 23(3) 524-534: $80422015000300524 \& \ln \mathrm{A}=\mathrm{en} \& n \mathrm{~m}=$ iso.

Guirro, E. \& Guirro, R. (2003). Fisioterapia Dermatofuncional: fundamentos, recursos, patologias. Barueri: Manole,

Lira, A. G., Ganen, A. P., Lodi, A. S., Alvarenga, M. S. (2017). Uso de redes sociais, influência da mídia e insatisfação com a imagem corporal de adolescentes brasileiras. J. bras. psiquiatr., 66(3), 164-171. http://www.scielo.br/scielo.php?script=sci_arttext\&pid=S0047$20852017000300164 \& \operatorname{lng}=$ en $\&$ nrm=iso.

Milani, G. B., João, S. M. A. \& Farah, E. A. (2006). Fundamentos da Fisioterapia dermato-funcional: revisão de literatura. Fisioterapia e Pesquisa, 13(1), 3743. http://www.periodicos.usp.br/fpusp/article/view/76159/79904.

Normando, D. (2012). A Estética e a Percepção Humana. Dental Press J Ortho, 17(5), 1. https://www.scielo.br/pdf/dpjo/v17n5/pt_01.pdf.

Oliveira, A. (2019). O que é Fisioterapia Dermatofuncional? Educa Mais Brasil. https://www.educamaisbrasil.com.br.

Pinheiro, T. A. (2020). Relação dos Procedimentos Estéticos com satisfação da Autoimagem Corporal e Autoestima de Mulheres. https://cathedral.ojs.galoa.com.br.

Russo, R. (2005) Imagem corporal: construção através da cultura do belo. Movimento e Percepção, Brasília. http://ferramentas.unipinhal.edu.br/movimentoepercepcao/viewarticle.php?id=39

SBCP. Sociedade Brasileira de Cirurgia Plástica. (2020). Líder Mundial. http://www2.cirurgiaplastica.org.br/blog/2020/02/13/lider-mundial/

SBD. Sociedade Brasileira de Dermatologia. (2019). o que é Dermatologia/?. http://www.sbd.org.br/dermatologia/sobre-a-dermatologia/o-que-edermatologia/.

Schmitz, D. S., Laurentino, L. \& Machado, M. (2012) Estética Facial e Corporal: uma revisão bibliográfica. Univali, 1-12. http://siaibib01.univali.br/pdf/Delourdes\%20Schafascheck\%20Schmitz,\%20Lucia\%20Laurentino.pdf. 